\title{
Monitoring of efficacy and safety of artemisinin- based anti-malarials for treatment of uncomplicated malaria: a review of evidence of implementation of anti-malarial therapeutic efficacy trials in Tanzania
}

Alex Shayo ${ }^{1}$, Joram Buza ${ }^{1}$ and Deus S Ishengoma ${ }^{2 *}$

\begin{abstract}
Background: Prompt diagnosis and effective treatment are considered the cornerstones of malaria control and artemisinin-based combination therapy (ACT) is currently the main anti-malarial drugs used for case management. After deployment of ACT due to widespread parasite resistance to the cheap and widely used anti-malarial drugs, chloroquine and sulphadoxine/pyrimethamine, the World Health Organization recommends regular surveillance to monitor the efficacy of the new drugs. The present paper assessed the implementation of anti-malarial efficacy testing for monitoring the therapeutic efficacy of ACT for treatment of uncomplicated malaria in Tanzania before and after policy changes in 2006.
\end{abstract}

Methods: A literature search was performed for published clinical trials conducted in Tanzania from 2001 to 2014 . It focused on studies which assessed at least one form of ACT for treatment of uncomplicated falciparum malaria in children less than 10 years and reported efficacy and safety of the tested anti-malarials. References were imported into the Endnote library and duplicates removed. An electronic matrix was developed in Microsoft Excel followed by full text review with predetermined criteria. Studies were independently assessed and information related to ACT efficacy and safety extracted.

Results: Nine papers were selected from 125 papers screened. The efficacy of both artemether-lumefantrine (AL) and artesunate-amodiaquine (AS $+\mathrm{AQ}$ ) against uncomplicated $P$. falciparum infections in Tanzania was high with PCRcorrected cure rates on day 28 of $91-100 \%$ and $88-93.8 \%$, respectively. The highest day-3 parasite positivity rate was $1.4 \%$. Adverse events ranged from mild to serious but were not directly attributed to the drugs.

Conclusion: ACT is efficacious and safe for treatment of uncomplicated malaria in Tanzania. However, few trials were conducted in Tanzania before and after policy changes in 2006 and thus more surveillance should be urgently undertaken to detect future changes in parasite sensitivity to ACT.

Keywords: Combination therapy, Artemether, Lumefantrine, Artesunate, Amodiaquine, Efficacy, Safety, Plasmodium falciparum and Tanzania

\footnotetext{
* Correspondence: deusishe@yahoo.com

${ }^{2}$ National Institute for Medical Research, Tanga Research Centre, P.O Box

5004, Tanga, Tanzania

Full list of author information is available at the end of the article
} 


\section{Background}

Malaria is by far the most important parasitic disease in Tanzania and in other tropical countries, causing loss of life and morbidity with more than three billion people at risk globally [1]. Prompt diagnosis and effective treatment are considered the cornerstones of malaria control [2-4]. However, resistance of malaria parasites to the cheap and commonly used anti-malarials has become a major challenge to malaria control. Worldwide resistance of Plasmodium falciparum to chloroquine (CQ) and the rapid spread of resistance to sulphadoxinepyrimethamine (SP) prompted the introduction of artemisinin combination therapy (ACT). In 2001, a World Health Organization (WHO) expert panel recommended use of ACT for treatment of uncomplicated falciparum malaria in all endemic countries [5].

The combination therapy involves simultaneous use of two or more blood schizonticidal drugs with independent modes of action and different biochemical targets in the parasite, a mechanism which delays development of parasite resistance [6,7]. ACT can be either fixed-combination medicinal products, in which different drugs are coformulated in the same tablets or capsules, or multiple drug therapy, in which the components are co-administered in separate tablets or capsules. The short-lived artemisininderivative component of ACT causes rapid and effective reduction of parasite biomass and gametocyte carriage, while the partner drug with a longer duration of action clears the remaining parasite biomass. The two drugs work together to achieve effective clinical and parasitological cure and protect each other from development of resistance by $P$. falciparum [5].

The current recommended combinations are artemetherlumefantrine $(\mathrm{AL})$, artesunate-amodiaquine $(\mathrm{AS}+\mathrm{AQ})$, artesunate-mefloquine (AS + MQ), dihydroartemisininpiperaquine (DHA $+\mathrm{PQ}$ ), and artesunate-sulphadoxine/ pyrimethamine $(\mathrm{AS}+\mathrm{SP})$ [8]. Artemisinin-naphthoquine combination $\left(\mathrm{ARCO}^{\mathrm{rm}}\right)$ has also been tested and has shown some potentials as a new generation ACT for the treatment of uncomplicated malaria, but it is still under further clinical evaluation [9]. Prior to policy changes, malaria-endemic countries had to choose among the above combinations based on different factors, such as price, level of parasite resistance to the partner drug in the local parasite population and the capacity of the country to sustainably supply the anti-malarials [8,10-12]. Thus, $\mathrm{AL}$ and $\mathrm{AS}+\mathrm{AQ}$ are the main ACT that have been widely deployed in majority of the African countries [13].

Parasite resistance to anti-malarials is of great concern in the efforts to control malaria worldwide. The parasites develop resistance by initially becoming tolerant to the drugs before they become fully resistant. The resistant parasites have an ability to survive under therapeutic levels of anti-malarial drugs which would otherwise kill both sensitive and tolerant parasites [14]. Parasite resistance to the anti-malarials can be assessed through in vivo, in vitro tests, analysis of known molecular markers of parasite resistance and by measurement of drug levels among patients treated with the respective anti-malarials [15-18]. Measures such as parasite clearance time, fever clearance time or gametocyte clearance time in in vivo and in vitro assays are used to indirectly detect any variation in parasite sensitivity thereby facilitating early warning in case of emergence of tolerance or resistance [19-21].

In vivo response of patients to treatments provides more information to clinicians and policy makers and is considered the gold standard for assessing anti-malarial efficacy. However, therapeutic efficacy must be interpreted as an interaction between the host factors (e.g., age, immunity and pharmacogenetics), the parasite factors (e.g., biomass, resistance) and the drug factors (e.g., pharmacokinetic properties, drug quality etc.) [17]. In vitro and molecular studies on the other hand, are useful in providing addition information on the parasite susceptibility without confounding effects of host factors although it is difficult to judge their clinical relevance [17]. It is only recently that K13-propeller polymorphism has been documented as a molecular marker for monitoring artemisinin resistance [22] and simpler genotyping protocols will be required before it can be fully adopted as a routine surveillance tool in most endemic countries with limited technical and infrastructural resources. Thus, effective monitoring of ACT has been and will continue to rely largely on in vivo studies with adequate follow-up. However, in vivo efficacy studies have logistics and cost implications which have limited their regular implementation.

WHO recommends regular efficacy testing for monitoring the efficacy of anti-malarials $[2,8,23]$. In Tanzania, the national malaria control programme (NMCP), in collaboration with its partners, including research institutions, medical universities, WHO country office and others, including funding agencies, have been conducting regular therapeutic efficacy trials (TETs). The efforts of the NMCP to ensure regular TETs have also been complemented by trials conducted by independent researchers. Thus, regular implementation of TETs is one of the priority activities of the Tanzania NMCP, which provides useful data for monitoring the efficacy of ACT and detecting emergence of drug tolerance/resistance to these and other anti-malarials used in the country. The findings of these studies have been used to guide the NMCP in reviewing and changing anti-malarial drug policy in the past $[24,25]$.

Tanzania changed its malaria treatment policy from CQ to SP monotherapy as the first-line drug for the treatment of uncomplicated malaria in 2001 [25]. However, shortly after its introduction, $P$. falciparum resistance to SP was reported [26,27] and this forced the 
country to change the guidelines in 2006 [24] to introduce ACT. Studies conducted in 2004 in Tanzania indicated that the mean SP treatment failure was as high as 25.5\% [28] which was higher than the WHO recommended cut-off failure rate (15\%), above which policy changes have to be made [2,29]. Whereas Zanzibar adopted AS + AQ as first-line treatment in November 2001 [30], Tanzania mainland adopted AL as first-line anti-malarial treatment of uncomplicated falciparum malaria in November 2006 and became fully rolled out in January 2007 [24].

Unfortunately, artemisinin-resistant field isolates have been reported recently in four countries of Southeast Asia (Cambodia, Myanmar, Thailand, and Vietnam) and threatens the current progress in controlling the disease $[1,2,19]$. There is a potential for such isolates to spread to other malaria-endemic regions, including sub-Saharan countries (SSA) as happened with previous anti-malarials [31,32], and such parasites might be extremely difficult to control. Such threat underscores the importance of intensive surveillance of artemisinin resistance to prevent the spread of resistance to other countries, as recommended within the WHO Global Plan for artemisinin resistance containment (GPARC) [23]. Such surveillance facilitates early detection of emergence and spread of tolerance/resistance to ACT and provides evidence for formulating mitigation and containment strategies as recommended by WHO $[23,33]$, thus helping to safeguard the long-term usefulness of these drugs.

The present paper reviewed the implementation of in vivo efficacy testing in Tanzania before and after deployment of ACT in order to monitor the efficacy of ACT for the treatment of uncomplicated malaria. The paper compares the cure rates, parasite clearance and fever clearance times and safety data reported in clinical trials involving ACT in Tanzania that were published between 2001 and 2014. It provides updates on country-specific performance of ACT after its widescale deployment for treating uncomplicated falciparum malaria.

\section{Methods}

Published literature was searched and it involved papers published from January 2001 to August 2014. English language articles indexed in PubMed were searched using search terms: 'Tanzania AND malaria AND artemether-lumefantrine,' 'Tanzania AND malaria AND artesunate-amodiaquine, 'Tanzania AND malaria AND artesunate-mefloquine,' 'Tanzania AND malaria AND dihydroartemisinin-piperaquine' and 'Tanzania AND malaria AND artesunate-sulphadoxine-pyrimethamine'. PubMed was used for primary search but in addition, Google Scholar, the Worldwide Antimalarial Resistance Network (WWARN) standardized analyses of ACT efficacy data repository and the African Journals Online (AJOL) were used to confirm that no study was missed. Inclusion criteria were clinical trials conducted in Tanzania between 2001 and 2014 and involved at least one ACT for treatment of uncomplicated falciparum malaria. The studies should have reported the efficacy and/or safety of the tested drugs. The starting year (i.e., 2001) was purposely chosen because that was the year when WHO advocated use of ACT for treating uncomplicated falciparum malaria [5]. References were imported into the Endnote library and duplicates removed. An electronic matrix was developed in Microsoft Excel followed by full text review with predetermined criteria. The selected studies were each given an identification number, independently assessed for key information on efficacy and safety of ACT, which was extracted and summarized in tables and texts.

\section{Results}

Literature search yielded 126 records, and 21 of these were duplicate records which were removed. The titles and abstracts of the remaining records (105) were screened based on the inclusion criteria and 11 articles qualified for a full-text review. From the review, two additional papers were from multicentre trials across Africa, which partly included Tanzania, and these were also removed. Only nine papers were left and fully reviewed as summarized in Figure 1.

\section{Studies conducted to test the efficacy of ACT in Tanzania} Before and after the official adoption of ACT for treatment of uncomplicated malaria in Tanzania in 2007 [24], nine clinical trials were conducted in the country to assess the efficacy and/or safety of ACT for treatment of uncomplicated falciparum malaria (Table 1). All except three studies were conducted in the eastern part of Tanzania, with only one study from Zanzibar (Figure 2). Of the nine studies, three were conducted before the official adoption of ACT in Tanzania while the other six were undertaken thereafter (Table 1). All of the nine trials included AL testing, while five of these tested AL alone [34-38], three (37.5\%) tested AL with AS + AQ $[30,39,40]$, and one trial tested $\mathrm{AL}$ with azithromycin (AZ) [41]. Six of the trials that tested AL were randomized trials with more than one arm (Table 1).

\section{Treatment outcome in studies that reported the efficacy of ACT in Tanzania}

For the trials that tested AL, the reported PCR-corrected cure rates ranged from 91 to $100 \%$ (Table 2). The highest cure rate $(100 \%)$ was reported by the studies conducted in Kyela in 2004, Tabora in 2011 and Muheza in 2013, while the lowest (91\%) was reported in Muheza in 2008. PCRcorrected cure rates of $\mathrm{AS}+\mathrm{AQ}$ ranged from 88.8 to 


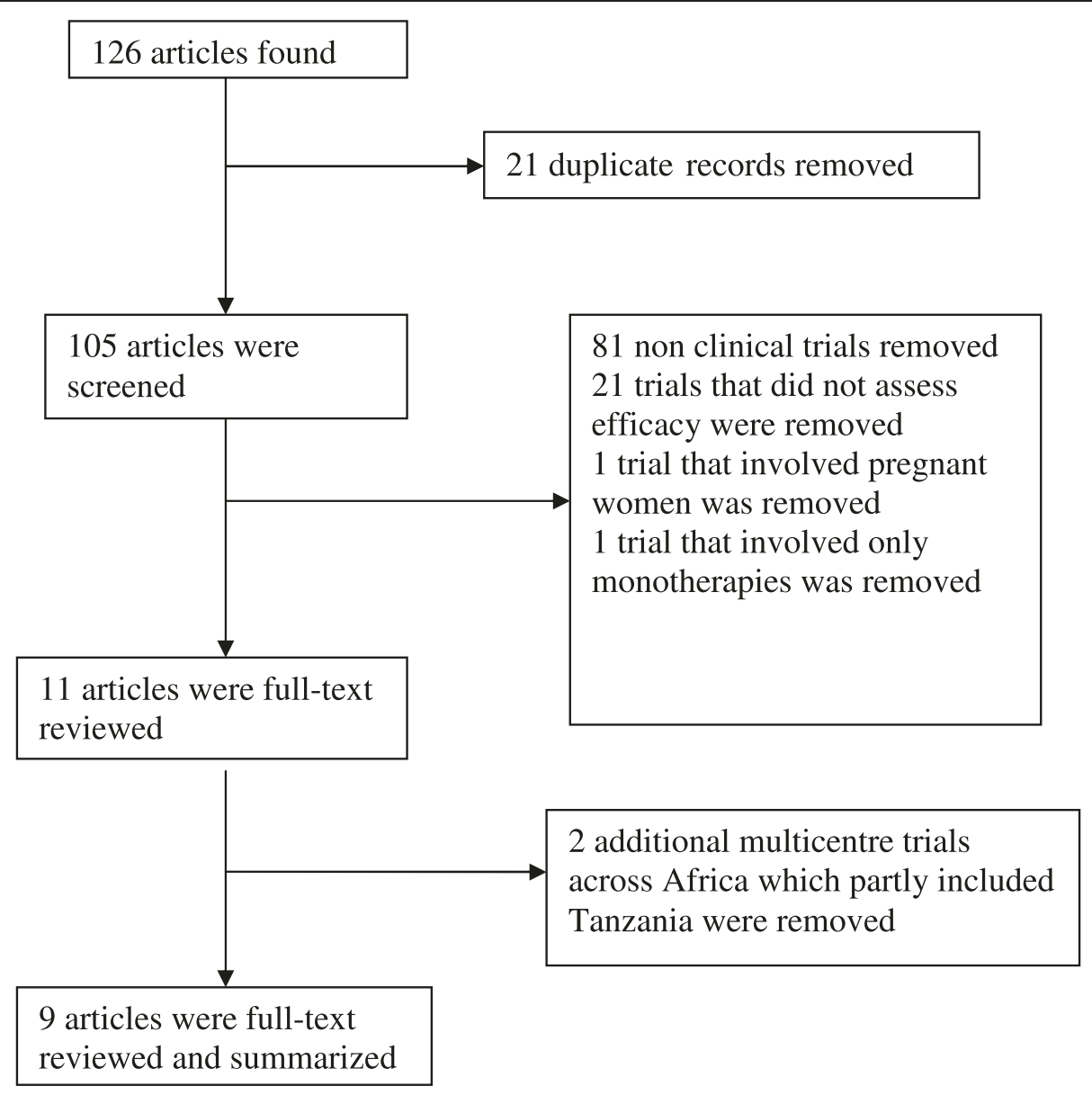

Figure 1 Flow diagram for the literature search

93.8\% (Table 2). The PCR-corrected cure rate of AZ + AS was $68 \%$. For the studies that tested AL when administered under supervision or unsupervised, the cure rates were comparable in the two groups. Although the study that tested the efficacy of AL when given unsupervised reported that more than half of the patients had recurrent infections within the 42-day follow-up period, the majority of these were due to re-infections and the cure rate was not significantly different when compared to the supervised arm. Studies that compared the efficacy of AL and AS + AQ showed a significantly lower risk of re-infection after treatment with AL than after treatment with AS + AQ [30,40].

Fever, parasite and gametocyte clearance in studies that reported efficacy of ACT in Tanzania

Among the five supervised trials that reported fever clearance, more than 80 and $79 \%$ of the patients cleared fever by day 1 post-treatment with AL and AS + AQ, respectively. None of the patients had fever on day 3 (Table 3). Among the five studies that reported parasite clearance, two studies showed day 3 parasitaemic cases of 1.1 and $1.4 \%$ (Table 3).

In the present review, four papers reported gametocyte carriage $[30,34,37,40]$ and the proportion of patients with gametocytes was significantly reduced from that recorded during enrolment compared to what was reported after treatment with AL or AS + AQ. Joseph et al. [34] showed an unusual increase in gametocytes in one patient treated with AL, from four on day-0 to 68 sexual parasites per 500 leucocytes on day- 2 .

\section{Day-7 plasma lumefantrine levels}

Three studies measured the median day-7 lumefantrine levels $[34,36,37]$ and one of these showed that the median plasma lumefantrine concentration was significantly higher in the supervised than in the unsupervised group $(\mathrm{P}<0.001)$ [35]. Furthermore, the median day-7 plasma lumefantrine concentration was significantly lower in patients with recrudescence compared to those with reinfections or no parasite re-appearance [38]. It was further shown that lumefantrine concentration at day 7 tended to 
Table 1 Study design and baseline characteristics of efficacy trials that assessed AL and AS + AQ

\begin{tabular}{|c|c|c|c|c|c|c|c|c|c|c|}
\hline Study ID & Authors & $\begin{array}{l}\text { Year } \\
\text { of } \\
\text { study }\end{array}$ & $\begin{array}{l}\text { Study } \\
\text { duration in } \\
\text { months }\end{array}$ & $\begin{array}{l}\text { Study } \\
\text { site }\end{array}$ & Randomization & $\begin{array}{l}\text { Sample } \\
\text { size }\end{array}$ & $\begin{array}{l}\text { Days of } \\
\text { follow- } \\
\text { up }\end{array}$ & $\begin{array}{l}\text { Parasitaemia } \\
\text { inclusion } \\
\text { criteria/ } / \mu \mathrm{l}\end{array}$ & $\begin{array}{l}\text { Pf- } \\
\text { GMPD }\end{array}$ & Supervised \\
\hline TZ001_AL & $\begin{array}{l}\text { Martensson } \\
\text { et al. [31] }\end{array}$ & $\begin{array}{l}2002- \\
2003\end{array}$ & 4 & Zanzibar & Yes, two arms & 200 & 42 & $2,000-200,000$ & 13,731 & Yes \\
\hline $\begin{array}{l}\text { TZ001_AS } \\
+\mathrm{AQ}\end{array}$ & $\begin{array}{l}\text { Martensson } \\
\text { et al. [31] }\end{array}$ & $\begin{array}{l}2002- \\
2003\end{array}$ & 4 & Zanzibar & Yes, two arms & 208 & 42 & $2,000-200,000$ & 19,731 & Yes \\
\hline TZ002_AL & $\begin{array}{l}\text { Mutabingwa } \\
\text { et al. [40] }\end{array}$ & $\begin{array}{l}2002- \\
2004\end{array}$ & 26 & Muheza & Yes, four arms & 519 & 28 & $\geq 2,000$ & 19,280 & No \\
\hline $\begin{array}{l}\text { TZO02_AS } \\
+\mathrm{AQ}\end{array}$ & $\begin{array}{l}\text { Mutabingwa } \\
\text { et al. [40] }\end{array}$ & $\begin{array}{l}2002- \\
2004\end{array}$ & 26 & Muheza & Yes, four arms & 515 & 28 & $\geq 2,000$ & 18,920 & No \\
\hline TZ003_AL & $\begin{array}{l}\text { Kabanywanyi } \\
\text { et al. [39] }\end{array}$ & 2004 & 7 & Kyela & Yes, four arms & 99 & 28 & $2,000-200,000$ & 43,115 & Yes \\
\hline $\begin{array}{l}\text { TZ003_AS + } \\
\text { AQ }\end{array}$ & $\begin{array}{l}\text { Kabanywanyi } \\
\text { et al. [39] }\end{array}$ & 2004 & 7 & Kilombero & Yes, four arms & 76 & 28 & $2,000-200,000$ & 49,348 & Yes \\
\hline TZ004_AL & $\begin{array}{l}\text { Sykes et al. } \\
{[41]}\end{array}$ & 2008 & 7 & Muheza & Yes, two arms & 132 & 42 & $2,000-200,000$ & $24,280^{* *}$ & Partial \\
\hline $\begin{array}{l}\text { TZO04_AZ + } \\
\text { AS }\end{array}$ & $\begin{array}{l}\text { Sykes et al. } \\
{[41]}\end{array}$ & 2008 & 7 & Muheza & Yes, two arms & 129 & 42 & $2,000-200,000$ & $20,960^{* *}$ & Partial \\
\hline TZO05_AL & $\begin{array}{l}\text { Ngasala et al. } \\
\text { [36] }\end{array}$ & $\begin{array}{l}2007- \\
2008\end{array}$ & 12 & Bagamoyo & Yes, two arms & 180 & 56 & $2,000-200,000$ & 41,885 & Yes \\
\hline TZO05*_AL & $\begin{array}{l}\text { Ngasala et al. } \\
{[36]^{*}}\end{array}$ & $\begin{array}{l}2007- \\
2008\end{array}$ & 12 & Bagamoyo & Yes, two arms & 179 & 56 & $2,000-200,000$ & 38,272 & No \\
\hline TZ006_AL & $\begin{array}{l}\text { Ngasala et al. } \\
\text { [37] }\end{array}$ & 2007 & 6 & Kibaha & No, single arm & 244 & 42 & $N R$ & 19,054 & No \\
\hline TZ007_AL & $\begin{array}{l}\text { Kamugisha } \\
\text { et al. [35] }\end{array}$ & $\begin{array}{l}2010- \\
2011\end{array}$ & 12 & Mwanza & No, single arm & 108 & 28 & $2,000-200,000$ & 5,608 & Yes \\
\hline TZ008_AL & $\begin{array}{l}\text { Joseph et al. } \\
\text { [34] }\end{array}$ & 2011 & 3 & Tabora & No, single arm & 20 & 28 & $2,000-200,000$ & 39,400 & Partial \\
\hline TZ009_AL ${ }^{* * *}$ & $\begin{array}{l}\text { Shayo et al. } \\
\text { [38] }\end{array}$ & 2013 & 2 & Muheza & No, single arm & 88 & 28 & $250-200,000$ & 18,603 & Yes \\
\hline
\end{tabular}

Doses of AL were given twice a day for three days; Doses of AS + AQ were given once a day for three days;

TZ005_AL was the same study split into supervised and unsupervised*arm; $N R=$ Not reported, Pf-GMPD $=P$. falciparum geometric mean parasite density of asexual parasites per microlitre of blood. ${ }^{* *}$ median parasite counts (asexual parasites per microlitre of blood) reported

***The study was conducted after malaria transmission had declined in the area and recruited children aged six months to 10 years and low cut-off of parasite density (250 asexual parasites/ $\mu \mathrm{l})$.

decrease with a unit increase in weight $(\mathrm{kg})$, although the decrement was not statistically significant [34].

\section{Safety profile of ACT reported in clinical trials conducted in Tanzania}

The safety of ACT was assessed by recording the nature and incidence of solicited and unsolicited adverse events and serious adverse events. An adverse event was defined as any undesirable medical occurrence (symptoms, signs or laboratory findings) in a patient during the study regardless of whether it was related to the treatment. Adverse events were judged according to their causal association with ACT (unlikely, possible and probable) and severity (mild, moderate or severe) [33]. Cough was the most common adverse event among children treated with AL, while severe malaria was the most reported serious adverse event requiring hospitalization among children treated with AL or AS + AQ (Table 4).
Other adverse events (as summarized in Table 4) were mild and not directly attributed to the treatment.

\section{Discussion}

Following the recent reports of emergence of P. falciparum artemisinin-resistant field isolates in Southeast Asia $[1,2,19]$ and the threat of such parasites spreading to other malaria-endemic countries, country-specific evidence based on reliable data are urgently required to monitor the efficacy of the drugs and support timely review and implementation of malaria treatment guidelines. Surveillance of anti-malarial efficacy is crucial to enable early detection of emergence of drug resistance when it happens before it spreads in most of the populations, as happened for CQ and SP [33]. Information generated from such surveillance provides evidence to relevant national and international authorities for policy formulation and review. This review was undertaken to assess the implementation of efficacy testing for 


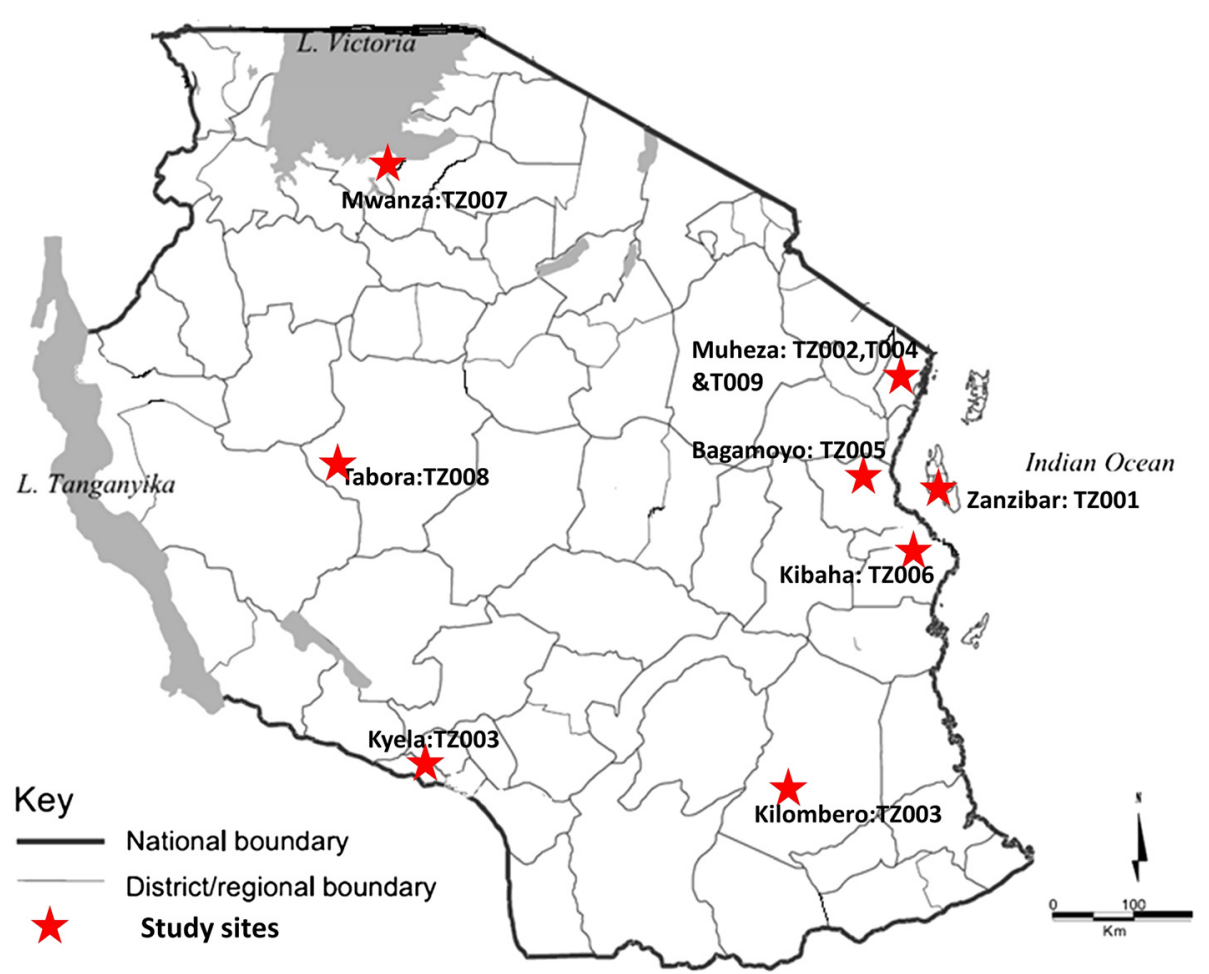

Figure 2 Map of Tanzania showing the location at which the reviewed studies were conducted.

Table 2 Treatment outcome reported in efficacy trials in Tanzania

\begin{tabular}{ll}
\hline Study ID & Day-28 PCR-corrected \% cure rate $\mathbf{( 9 5 \% ~ C l )}$ \\
\hline TZ001_AL & $97.0(\mathrm{NR})$ \\
TZ001_AS+AQ & $91.0(\mathrm{NR})$ \\
TZ002_AL & $97.2(\mathrm{NR})$ \\
TZ002_AS+AQ & $88.8(\mathrm{NR})$ \\
TZ003_AL & $100.0(\mathrm{NR})$ \\
TZ003_AS + AQ & $93.8(\mathrm{NR})$ \\
TZ004_AL & $91.0(\mathrm{NR})$ \\
TZ004_AZ+AS & $68.0(\mathrm{NR})$ \\
TZ005_AL & $98.8(95.5-99.7)$ \\
TZ005*AAL & $98.2(94.5-99.4)$ \\
TZ006_AL & $95.1(91.4-97.7)$ \\
TZ007_AL & $96.0(\mathrm{NR})$ \\
TZ008_AL & $100.0(\mathrm{NR})$ \\
TZ009_AL & $100.0(\mathrm{NR})$ \\
\hline
\end{tabular}

TZ005_AL was the same study split into supervised and unsupervised*arm. 95\% Cl=95\% Confidence interval;

$N R=$ Not reported. monitoring of therapeutic efficacy of ACT for treatment of uncomplicated falciparum malaria in Tanzania before and after policy changes (in 2006).

Apparently, due to limited resources, especially funding, NMCPs in most endemic countries have not been able to implement regular anti-malarial drug efficacy monitoring at sentinel sites and there has been a strong call for regional networks to facilitate the implementation [42]. The former East African Network for monitoring anti-malarial treatment (EANMAT) forged a partnership between the ministries of health and the research community in East African countries and facilitated monitoring of antimalarial drug resistance in the region [28]. However, EANMAT collapsed as a result of many factors, including dependence on short-term donor funding.

The findings of this review showed that nine clinical trials have been conducted to monitor the efficacy of ACT before and after Tanzania adopted ACT for treatment of uncomplicated malaria. Of these, only one study [39] was conducted within the in vivo efficacy testing framework of Tanzania NMCP/EANMAT with financial support from NMCP and EANMAT. This might be partly due to lack of funding, or due to complacency attributed to perceived high therapeutic efficacy of ACT. However, in Tanzania, NMCP-supported TETs have resumed since 2011, although the findings have not yet been published. 
Table 3 Fever and parasite clearance in efficacy trials in Tanzania

\begin{tabular}{|c|c|c|c|c|}
\hline Study ID & Fever clearance by D1 (\%) & Day 1 Parasitaemic (\%) & Day 2 Parasitaemic (\%) & Day 3 Parasitaemic (\%) \\
\hline TZO01_AS + AQ & 79.0 & 66.0 & 10.0 & 0 \\
\hline TZ001_AL & 67.0 & 83.0 & 10.0 & 0 \\
\hline TZO05_AL & 64.3 & 71.3 & 6.7 & 1.1 \\
\hline TZO07_AL & 95.0 & 32.0 & 11.7 & 0 \\
\hline TZO08_AL & 95.0 & NR & 0 & 0 \\
\hline TZ009_AL & 73.9 & 77.3 & 19.5 & 1.4 \\
\hline
\end{tabular}

D1refers to day 1;

$N R=$ Not reported

The present review has shown that the efficacy of AL, which is the first-line anti-malarial drug for treatment of uncomplicated falciparum malaria in Tanzania, was high even after unsupervised treatment. The PCR-corrected cure rate on day 28 was $>91 \%$ and this is in line with findings from other studies in eastern Africa [43,44]. The high cure rate $(100 \%)$ reported in Muheza in 2004 [39] prior to the official adoption of AL was similar to the cure rate reported in other African countries $[45,46]$. The efficacy of AS + AQ, which is currently the first-line anti-malarial drug for treatment of uncomplicated falciparum malaria in Zanzibar (an island part of the United Republic of Tanzania) [30], was also high although comparably lower than that of AL (PCR-corrected cure rate was $88.8-93.8 \%$ for AS + AQ compared to $91-100 \%$ for $\mathrm{AL})$. The lower cure rates of $\mathrm{AS}+\mathrm{AQ}$ compared to AL could be attributed to the fact that AQ had been extensively used in the country and was also adopted as a second-line anti-malarial drug together with SP (which was the first-line) in 2001 [25]. Since AQ resistance had been reported in Tanzania $[27,47]$, addition of an artemisinin to AQ was unlikely to make a combination with high therapeutic efficacy. Similar failure rates have been reported in other SSA countries that used AQ extensively prior to introduction of AS + AQ [48].

The cure rates of $\mathrm{AQ}+\mathrm{AS}$ in the present review compares well with those reported elsewhere in East Africa whereby day- 28 adequate clinical and parasitological response (ACPR) in children treated with AS + AQ was 90.2\% in Kenya [49], 90.3\% in Rwanda [50] and 91.7\% in Uganda [51]. These rates were comparatively lower than those of AL. However, AS + AQ was selected and is still being used as the first-line anti-malarial in some countries when other factors apart from efficacy were considered. In Burundi, prior to policy changes, day-14 ACPR in children treated with $\mathrm{AS}+\mathrm{AQ}$ was reported to be 95.3\% compared to $99.3 \%$ for AL. However, considering other factors such as acceptability by users, adherence

Table 4 Adverse events reported in efficacy trials in Tanzania

\begin{tabular}{|c|c|c|}
\hline Study ID & Adverse events reported & Comments \\
\hline $\begin{array}{l}\text { TZO01_AS } \\
+ \text { AQ }\end{array}$ & Severe malaria (3.4\%) & Not attributed to the treatment \\
\hline TZ001_AL & Severe malaria (1.0\%) & Not attributed to the treatment \\
\hline $\begin{array}{l}\text { TZOO2_ } \\
\text { AL }\end{array}$ & Death $(0.2 \%)$ & Not attributed to the treatment \\
\hline $\begin{array}{l}\text { TZOO4_ } \\
\text { AL }\end{array}$ & $\begin{array}{l}\text { Gastrointestinal complaints }(5.3 \%) \text {, vomiting }(1.5 \%) \text {, dermatological } \\
\text { (including itching) }(3.0 \%) \text {, respiratory (including respiratory infection) } \\
(15.9 \%) \text {, dizziness }(0.8 \%) \text {, convulsions ( } 2.3 \%)\end{array}$ & $\begin{array}{l}\text { Gastrointestinal complaints were likely to be attributed to the } \\
\text { drugs }\end{array}$ \\
\hline $\begin{array}{l}\mathrm{TZOO4} \\
\mathrm{AZ}+\overline{\mathrm{AS}}\end{array}$ & $\begin{array}{l}\text { Gastrointestinal complaints }(2 \%) \text {, vomiting }(7.6 \%) \text {, dermatological } \\
\text { (including itching) }(5.4 \%) \text {, respiratory tract respiratory infections } \\
(11.6 \%) \text {, dizziness }(1.6 \%) \text {, convulsions }(0.8 \%)\end{array}$ & $\begin{array}{l}\text { Gastrointestinal complaints were likely to be attributed to the } \\
\text { drugs }\end{array}$ \\
\hline $\begin{array}{l}\text { TZO05_ } \\
\text { AL }\end{array}$ & $\begin{array}{l}\text { Severe malaria }(4 \%) \text {, vomiting }(1 \%) \text {, cough }(10 \%) \text {, abdominal pain } \\
(1 \%) \text {, diarrhoea }(3 \%) \text {, weakness }(1 \%) \text {, upper respiratory tract } \\
\text { infections }(22 \%) \text {, skin infections }(9 \%) \text {, urinary tract infection }(5 \%) \text {, } \\
\text { otitis media }(4 \%) \text {, tonsillitis }(2 \%) \text {, conjunctivitis }(4 \%) \text {, worm } \\
\text { infestation }(1 \%) \text {, periodontitis }(1 \%) \text {, asthma }(0.3 \%)\end{array}$ & $\begin{array}{l}\text { Severe malaria was recorded as severe adverse event. The rest } \\
\text { were mild or moderate in severity }\end{array}$ \\
\hline $\begin{array}{l}\text { TZO06_ } \\
\text { AL }\end{array}$ & Severe malaria (1\%), fever (34\%), cough (34\%), diarrhoea (12\%) & $\begin{array}{l}\text { Severe malaria was recorded as severe adverse event. The rest } \\
\text { were mild or moderate in severity. None was considered related to } \\
\text { AL treatment }\end{array}$ \\
\hline $\begin{array}{l}\text { TZOO9_ } \\
\text { AL }\end{array}$ & $\begin{array}{l}\text { Cough (49.4\%), fever }(20.2 \%) \text {, abdominal pain }(10.1 \%) \text {, diarrhoea } \\
(1.3 \%) \text {, Headache }(1.3 \%) \text {, skin rashes }(1.3 \%)\end{array}$ & $\begin{array}{l}\text { No serious adverse events. All the AEs were not related to the } \\
\text { treatment }\end{array}$ \\
\hline
\end{tabular}


and cost, AS + AQ was chosen as the first-line anti-malarial for treatment of uncomplicated falciparum malaria in Burundi $[10,12]$. Similarly, Zanzibar adopted AS + AQ as the first-line anti-malarial despite lower day-28 ACPR compared to AL (97\% versus $91 \%$ for AS + AQ) [30].

There is a concern about the limited post-treatment prophylactic effects of both AL and AS + AQ in high transmission areas. In fact in one trial, more than half of the recruited patients had recurrent infections within the 42-day follow-up period after treatment with AL. However, the majority of recurrent infections were due to re-infections which suggests that the partner drug cannot give prolonged protection despite high therapeutic efficacy [36]. Similar high re-infections rates have been reported in other high transmission areas in Africa after AL treatment $[43,52]$. Studies that compared the efficacy of AL and AS + AQ showed a significantly lower risk of re-infection after treatment with AL compared to $\mathrm{AS}+\mathrm{AQ}[30,40]$ suggesting that AL confers a longer prophylactic effect than $\mathrm{AS}+\mathrm{AQ}$. The difference in prophylactic effect of the two drugs could be attributed to the longer half-life of lumefantrine compared to AQ. Thus, the concentration of the active amodiaquine metabolite might be lower or completely absent when a reinfection occurs compared to lumefantrine concentration. This observation has also been reported elsewhere in Africa where re-infection rates were higher after AS + AQ treatment than after AL $[48,53,54]$. However, a recent study has reported high level of resistance to lumenfatrine in the Democratic Republic of Congo [55] that threatens the therapeutic usefulness of AL and further monitoring is urgently needed in all malaria-endemic countries where AL is the first-line anti-malarial drug.

In most of the studies, a great majority of the recurrent infections were due to re-infections, when assessed with a step-wise PCR genotyping protocol, which signifies that the drugs are still efficacious and the high rates of re-infections could only be attributed to high malaria transmission. In terms of clinical practice, the high reinfection rates are of great concern among clinicians. Clinicians should be clearly guided on what to expect and how to handle such cases with recurrent infections within a period of three to eight weeks post-treatment. The observed high re-infection rates after ACT treatment underscores the importance of integrating treatment with vector control interventions, including use of long-lasting insecticide-treated nets so as to effectively block malaria transmission and prevent recurrent infections [56].

The study which tested AZ + AS showed that the drug had low efficacy ( 28 days ACPR $=68 \%$ ) and could not be considered a potential anti-malarial drug in Tanzania and other malaria-endemic countries [41]. It is plausible that since AZ is a common antibiotic in the treatment of trachoma, the local parasites might have been exposed to the drug leading to development of resistance [57]. This could have possibly compromised the efficacy of $\mathrm{AZ}+\mathrm{AS}$ combination. An alternative explanation for the observed lower efficacy of AZ + AS compared to adults in Asia is that the effective dose of AZ absorbed in often-malnourished African children might not be sufficient to achieve adequate cure rates. Malnourishment is known to reduce drug absorption [8] and cure rates among patients treated with different drugs. Furthermore, a recent review of $\mathrm{AZ}$ across continents for treating uncomplicated malaria revealed that $\mathrm{AZ}$ has low efficacy as a monotherapy for treatment of uncomplicated malaria and when used in combinations with other anti-malarials, it may need to be used at high doses which may affect tolerability to the drug [58].

Measurement and reporting of parasite clearance on day 3 after treatment with ACT is particularly important, as this is one of the first signals of emergence of parasite tolerance/resistance to artemisinin [23]. In the present review, two studies reported day-3 parasitaemic cases of 1.1 and $1.4 \%$ after treatment with AL [36,38] and the day 3 parasite positivity rates were lower than what has been previously reported [21]. However, the parasite positivity rate reported on day 2 in one of the studies conducted in Muheza district with moderate malaria transmission was higher than the rates reported in previous studies [21]. Thus, more studies will be required to confirm these findings and their role in possible emergence of artemisinin resistance. Although the proportion of patients with detectable parasitaemia on day 3 serves as a simple measure of parasite clearance time at the population level [21], it is often influenced by the baseline parasite density and the timing of parasite sampling, which can vary within and across studies. On the contrary, parasite clearance half-life doesn't depend on baseline parasite density and is thus considered a more reliable indicator of changes in parasite susceptibility to artemisinin. Measurement of parasite clearance at six-, eight- or 12-hourly intervals for the first 72 hours, as it is currently recommended $[59,60]$, provides a population level profile and useful data of parasite sensitivity to artemisinin. More accurate estimates of parasite clearance through frequent parasite counts are recommended $[59,60]$. However, the studies reviewed in this article were based on 24-hour sampling, which is not the recommended method for assessing parasite clearance and detection of tolerance/resistance to artemisinins.

Artemisinins are known to be highly potent anti-malarial drugs that are active against immature gametocytes and are useful in the reduction of malaria transmission and elimination/eradication agenda [61]. In clinical trials reviewed in this paper, it was shown that in fact $\mathrm{AL}$ and $\mathrm{AS}+\mathrm{AQ}$ have potentials to reduce gametocyte carriage 
$[30,34,37,40]$. However, the unusual increase in gametocytes from four on day- 0 to 68 sexual parasites per 500 leucocytes on day- 2 post AL treatment, as reported in one of the trials [34], needs to be further evaluated in the light of changes in the parasite sensitivity to ACT. Gametocyte clearance by ACT has also been documented by other studies in East Africa [62] and elsewhere [63,64] where significant reduction of gametocytes by day 14 after treatment with AL or AS + AQ was observed, indicating the potential advantages of ACT over non-artemisinin-based antimalarials.

It is well established that the efficacy of AL combination is strongly influenced by variations in the pharmacokinetics of lumefantrine among individuals [8]. The maximum therapeutic cure rate is achieved when the plasma drug concentration is adequately available for at least six days [65]. Measurements of day-7 plasma lumefantrine levels are particularly important in unsupervised trials as a measure of adherence to treatment, rather than the use of questionnaires [66]. Day-7 lumefantrine concentrations were significantly lower in unsupervised patients suggesting lower adherence to the drug dosage or fat intake advice. However these differences did not affect the cure rates and high therapeutic efficacy was achieved even in the unsupervised group, indicating that the parasites are highly susceptible to lumefantrine. The observation by other studies in East Africa which showed the median lumefantrine levels were significantly lower in unsupervised patients, but without any effects on the cure rates $[67,68]$, lends support to the findings of this review. It is clear that a high day-28 AL cure rate can be achieved despite low plasma lumefantrine levels, even among unsupervised patients. However care should be taken to avoid exposure of parasites to sub-therapeutic levels of the drugs and creating favourable conditions for emergence of lumefantrine resistance [69]. Monitoring of lumefantrine tolerance/resistance should also be implemented in order to safeguard usefulness of AL.

The present review showed no unexpected adverse events and overall, AL, AS + AQ and AZ + A were well tolerated. Admittedly, the few studies that reported safety profile in the present review (e.g., only one study reported safety data on AS + AQ while the rest reported AL safety) would not enable a firm comparison of safety of different anti-malarials. However, other studies in Africa have shown that certain mild or moderate adverse events, such as vomiting and anaemia, were more frequent in patients treated with AS + AQ than those treated with AL $[70,71]$. This review has shown that respiratory infection, including cough, was the most frequent adverse event in children treated with AL. This is in line with previous findings which showed that respiratory infections were common in African children with malaria $[72,73]$. The findings from a recent review on the safety of AL with other ACT in children [73] showed that adverse events were attributed to symptoms or progression of malaria and not directly to the drugs, and this lends support to the findings of this review. Thus, AL and AS + AQ are safe when used for treatment of uncomplicated malaria.

\section{Limitation of the review}

This review was meant to assess the implementation of the WHO recommendations of undertaking regular monitoring of antimalarial efficacy studies and also provide Tanzania-specific current efficacy and safety profile of ACT in the treatment of uncomplicated falciparum malaria. The review was limited to peer-reviewed publications, thus unpublished data were not included. However the review highlighted the levels of implementation of TETs in Tanzania and provides an overall country-specific performance of ACT after their wide-scale deployment as first-line anti-malarials for treating uncomplicated $P$. falciparum malaria in the country.

\section{Future studies}

Following the emergence of artemisinin resistance in Southeast Asia, manifested as delayed clearance of $P$. falciparum after treatment with artemisinins, the demand for tracking parasite sensitivity to artemisinin and its derivatives has become more important. More accurate estimates of parasite clearance measurements through frequent parasite counts (at least twice daily) to assess delayed parasite clearance should be adopted in future therapeutic efficacy testing studies [59,60]. However these changes have significant cost and logistic implications that must be addressed.

Given the recent documentation of K13-propeller polymorphism as a molecular marker for monitoring resistance of artemisinin and its derivatives [22] and despite absence of Asian mutant genotypes in SSA [74], future efficacy studies should incorporate assessment of this marker as a tool to track parasite tolerance or any changes in parasite sensitivity to ACT [75]. Furthermore, the recent report of high resistance to lumefantrine should also be assessed in both in vivo and in vitro studies. More importantly, optimization and testing of other methods for resistance surveillance such as ex-vivo and ring stage assay should be considered for future studies in SSA.

\section{Conclusion}

The present review has shown that few studies have been conducted in Tanzania to monitor the efficacy and safety of ACT and majority of these were not done under the NMCP framework. However, the findings revealed that the efficacy AL and AS + AQ was reasonably high and the drugs were safe when used for treatment of 
uncomplicated $P$. falciparum infections in Tanzania. These findings support continued use of AL and AS + AQ for the treatment of uncomplicated malaria in Tanzania mainland and Zanzibar, respectively. Although currently there is no evidence of artemisinin resistance in Africa, regular monitoring and surveillance, as recommended by the WHO-supported GPARC must be implemented so that the emergence of artemisinin resistance in African can be timely detected, reported and contained. More surveillance and monitoring of antimalarial efficacy and safety should be performed to detect future changes in parasite sensitivity to ACT.

\section{Abbreviations}

AS + AZ: Artesunate azithromycin; ACT: Artemisinin combination therapy; AE: Adverse event; AL: Artemether-lumefantrine; AS + AQ: Artesunateamodiaquine; SAE: Serious adverse event; CQ: Chloroquine; SP: Sulphadoxine/pyrimethamine; DHA + PQ: Dihydroartemisinin-piperaquine; AS + SP: Artesunate-sulphfadoxine/pyrimethamine; NMCP: National malaria control programme; TETs: Therapeutic efficacy trials; SSA: Sub-Saharan Africa; GPARC: Global plan for artemisinin resistance containment; EANMAT: East African network for monitoring anti-malarial treatment; WHO: World Health Organization.

\section{Competing interests}

The authors declare that they have no competing interests.

\section{Authors' contributions}

AS, JB and DSI conceived of the idea, AS performed the review and AS and DSI wrote the manuscript. All authors read, and approved the final version of the manuscript.

\section{Acknowledgements}

The authors would like to thank Dr Samuel Gesase for his critical review of the manuscript and the Tanzania Commission for Science and Technology through the Nelson Mandela African Institution of Science and Technology, for funding the study. Permission to publish this paper was obtained from the Director General of the National Institute for Medical Research.

\section{Author details}

'The Nelson Mandela African Institution of Science and Technology, P.O Box 447, Arusha, Tanzania. ${ }^{2}$ National Institute for Medical Research, Tanga Research Centre, P.O Box 5004, Tanga, Tanzania.

Received: 29 November 2014 Accepted: 15 March 2015 Published online: 29 March 2015

\section{References}

1. WHO. World Malaria Report. Geneva, Switzerland: World Health Organization; 2013.

2. WHO. Global Report on Antimalarial Drug Efficacy and Drug Resistance 2000-2010. Geneva, Switzerland: World Health Organization; 2010.

3. Assefa A, Kassa M, Tadese G, Mohamed H, Animut A, Mengesha T. Therapeutic efficacy of artemether/lumefantrine (Coartem) against Plasmodium falciparum in Kersa, South West Ethiopia. Parasit Vectors. 2010;3:1.

4. WHO. Malaria control today: current WHO recommendations. Geneva, Switzerland: World Health Organization; 2005.

5. WHO. Antimalarial drug combination therapy. Report of a WHO Technical Consultation. Geneva, Switzerland: World Health Organization; 2001

6. Hong NV, Amambua-Ngwa A, Tuan NQ, Cuong DD, Giang NTH, Dung NV, et al. Severe malaria not responsive to artemisinin derivatives in man returning from Angola to Vietnam. Emerg Infect Dis. 2014;20:1199-202.

7. Duffy PE, Sibley $\mathrm{CH}$. Are we losing artemisinin combination therapy already? Lancet. 2005;366:1908-9.

8. WHO. Guidelines for the treatment of malaria, Second edition. Geneva, Switzerland: World Health Organization; 2010.
9. Hombhanje FW, Linge D, Saweri A, Kuanch C, Jones R, Toraso S, et al. Artemisinin-naphthoquine combination (ARCO) therapy for uncomplicated falciparum malaria in adults of Papua New Guinea: a preliminary report on safety and efficacy. Malar J. 2009;8:196.

10. WHO. Guidelines for the treatment of malaria. Geneva, Switzerland: World Health Organization; 2006.

11. Amin AA, Zurovac D, Kangwana BB, Greenfield J, Otieno DN, Akhwale WS, et al. The challenges of changing national malaria drug policy to artemisinin-based combinations in Kenya. Malar J. 2007;6:72.

12. Ndayiragije A, Niyungeko D, Karenzo J, Niyungeko E, Barutwanayo M, Ciza A, et al. Efficacy of therapeutic combinations with artemisinin derivatives in the treatment of non complicated malaria in Burundi. Trop Med Int Health. 2004;9:673-9.

13. Bosman A, Mendis KN. A major transition in malaria treatment: the adoption and deployment of artemisinin-based combination therapies. Am J Trop Med Hyg. 2007;77(6 Suppl):193-7

14. Hastings IM, Watkins WM. Tolerance is the key to understanding antimalarial drug resistance. Trends Parasitol. 2006;22:71-7.

15. Bacon DJ, Jambou R, Fandeur T, Le Bras J, Wongsrichanalai C, Fukuda MM, et al. World Antimalarial Resistance Network (WARN) II: in vitro antimalarial drug susceptibility. Malar J. 2007;6:120.

16. Plowe CV, Roper C, Barnwell JW, Happi CT, Joshi HH, Mbacham W, et al. World Antimalarial Resistance Network (WARN) III: molecular markers for drug resistant malaria. Malar J. 2007;6:121.

17. Price RN, Dorsey G, Ashley EA, Barnes Kl, Baird JK, d'Alessandro U, et al. World Antimalarial Resistance Network I: clinical efficacy of antimalarial drugs. Malar J. 2007:6:119.

18. Barnes Kl, Lindegardh $\mathrm{N}$, Ogundahunsi O, Olliaro P, Plowe CV, Randrianarivelojosia $\mathrm{M}$, et al. World antimalarial resistance network (WARN) IV: Clinical pharmacology. Malar J. 2007;6:122.

19. Dondorp AM, Nosten F, Yi P, Das D, Phyo AP, Tarning J, et al. Artemisinin resistance in Plasmodium falciparum malaria. N Engl J Med. 2009;361:455-67.

20. Sowunmi A, Adewoye EO, Gbotsho GO, Happi CT, Sijuade A, Folarin OA, et al. Factors contributing to delay in parasite clearance in uncomplicated falciparum malaria in children. Malar J. 2010;9:53.

21. Stepniewska K, Ashley E, Lee SJ, Anstey N, Barnes Kl, Binh TQ, et al. In vivo parasitological measures of artemisinin susceptibility. J Infect Dis. 2010;201:570-9

22. Ariey F, Witkowski B, Amaratunga C, Beghain J, Langlois A-C, Khim N, et al. A molecular marker of artemisinin-resistant Plasmodium falciparum malaria. Nature. 2014;505:50-5.

23. WHO. Global plan for artemisinin resistance containment (GPARC). Geneva, Switzerland: World Health Organization; 2011.

24. MHSW. National Guidelines for malaria diagnosis and treatment. Ministry of Health: United Republic of Tanzania; 2006.

25. MoH. National Guidelines for malaria diagnosis and treatment. United Republic of Tanzania: Ministry of Health; 2000.

26. Mugittu K, Ndejembi M, Malisa A, Lemnge M, Premji Z, Mwita A, et al. Therapeutic efficacy of sulfadoxine-pyrimethamine and prevalence of resistance markers in Tanzania prior to revision of malaria treatment policy: Plasmodium falciparum dihydrofolate reductase and dihydropteroate synthase mutations in monitoring in vivo resistance. Am J Trop Med Hyg. 2004;71:696-702.

27. EANMAT. The efficacy of antimalarial monotherapies, sulphadoxinepyrimethamine and amodiaquine in East Africa: implications for sub-regional policy. Trop Med Int Health. 2003;8:860-7.

28. EANMAT. Monitoring antimalarial drug resistance within National Malaria Control Programmes: The EANMAT experience. Trop Med Int Health. 2001;6:891-8.

29. Kitua AY. Antimalarial drug policy: making systematic change. Lancet. 1999:354:siv32.

30. Mårtensson A, Strömberg J, Sisowath C, Msellem MI, Gil JP, Montgomery SM, et al. Efficacy of artesunate plus amodiaquine versus that of artemetherlumefantrine for the treatment of uncomplicated childhood Plasmodium falciparum malaria in Zanzibar, Tanzania. Clin Infect Dis. 2005:41:1079-86.

31. Mita T, Venkatesan M, Ohashi J, Culleton R, Takahashi N, Tsukahara T, et al. Limited geographical origin and global spread of sulfadoxine-resistant dhps alleles in Plasmodium falciparum populations. J Infect Dis. 2011;204:1980-8.

32. Roper C, Pearce R, Nair S, Sharp B, Nosten F, Anderson T. Intercontinental spread of pyrimethamine-resistant malaria. Science. 2004;305:1124.

33. WHO. Methods for surveillance of antimalaria drug efficacy. Geneva, Switzerland: World Health Organization; 2009. 
34. Joseph D, Kabanywanyi AM, Hulser R, Premii Z, Minzi OM, Mugittu K. Exploration of in vivo efficacy of artemether-lumefantrine against uncomplicated Plasmodium falciparum malaria in under fives in Tabora region. Tanzania Malar J. 2013;12:60.

35. Kamugisha E, Jing S, Minde M, Kataraihya J, Kongola G, Kironde F, et al. Efficacy of artemether-lumefantrine in treatment of malaria among under-fives and prevalence of drug resistance markers in lgombe-Mwanza, north-western Tanzania. Malar J. 2012;11:58.

36. Ngasala BE, Malmberg M, Carlsson AM, Ferreira PE, Petzold MG, Blessborn D, et al. Efficacy and effectiveness of artemether-lumefantrine after initial and repeated treatment in children $<5$ years of age with acute uncomplicated Plasmodium falciparum malaria in rural Tanzania: a randomized trial. Clin Infect Dis. 2011;52:873-82.

37. Ngasala BE, Malmberg M, Carlsson AM, Ferreira PE, Petzold MG, Blessborn D, et al. Effectiveness of artemether-lumefantrine provided by community health workers in under-five children with uncomplicated malaria in rural Tanzania: an open label prospective study. Malar J. 2011;10:64.

38. Shayo A, Mandara Cl, Shahada F, Buza J, Lemnge MM, Ishengoma DS. Therapeutic efficacy and safety of artemether-lumefantrine for the treatment of uncomplicated falciparum malaria in North-Eastern Tanzania. Malar J. 2014;13:376

39. Kabanywanyi AM, Mwita A, Sumari D, Mandike R, Mugittu K, Abdulla S. Efficacy and safety of artemisinin-based antimalarial in the treatment of uncomplicated malaria in children in southern Tanzania. Malar J. 2007;6:146.

40. Mutabingwa TK, Anthony D, Heller A, Hallett R, Ahmed J, Drakeley C, et al. Amodiaquine alone, amodiaquine + sulfadoxine-pyrimethamine, amodiaquine + artesunate, and artemether-lumefantrine for outpatient treatment of malaria in Tanzanian children: a four-arm randomised effectiveness trial. Lancet. 2005;365:1474-80.

41. Sykes A, Hendriksen I, Mtove G, Mandea V, Mrema H, Rutta B, et al. Azithromycin plus artesunate versus artemether-lumefantrine for treatment of uncomplicated malaria in Tanzanian children: a randomized, controlled trial. Clin Infect Dis. 2009;49:1195-201.

42. Talisuna AO, Karema C, Ogutu B, Juma E, Logedi J, Nyandigisi A, et al. Mitigating the threat of artemisinin resistance in Africa: improvement of drug-resistance surveillance and response systems. Lancet Infect Dis. 2012;12:888-96.

43. Bukirwa H, Yeka A, Kamya MR, Talisuna A, Banek K, Bakyaita N, et al. Artemisinin combination therapies for treatment of uncomplicated malaria in Uganda. PLoS Clin Trials. 2006;1:e7.

44. Juma EA, Obonyo CO, Akhwale WS, Ogutu BR. A randomized, open-label, comparative efficacy trial of artemether-lumefantrine suspension versus artemether-lumefantrine tablets for treatment of uncomplicated Plasmodium falciparum malaria in children in western Kenya. Malar J. 2008;7:190.

45. Guthmann J-P, Cohuet S, Rigutto C, Fortes F, Saraiva N, Kiguli J, et al. High efficacy of two artemisinin-based combinations (artesunate + amodiaquine and artemether + lumefantrine) in Caala, Central Angola. Am J Trop Med Hyg. 2006;75:143-5.

46. Falade C, Ogundele A, Yusuf B, Ademowo O, Ladipo S. High efficacy of two artemisinin-based combinations (artemether-lumefantrine and artesunate plus amodiaquine) for acute uncomplicated malaria in Ibadan, Nigeria. Trop Med Int Health. 2008;13:635-43.

47. Lemnge M, Alifrangis M, Kafuye MY, Gesase S, Minja D, Massaga JJ, et al. High reinfection rate and treatment failures in children treated with amodiaquine for falciparum malaria in Muheza villages, Northeastern Tanzania. Am J Trop Med Hyg. 2006;75:188-93.

48. Zwang J, Olliaro P, Barennes H, Bonnet M, Brasseur P, Bukirwa H, et al. Efficacy of artesunate-amodiaquine for treating uncomplicated falciparum malaria in sub-Saharan Africa: a multi-centre analysis. Malar J. 2009;8:203.

49. Thwing J, Odero C, Odhiambo F, Otieno K, Kariuki S, Ord R, et al. In vivo efficacy of amodiaquine-artesunate in children with uncomplicated Plasmodium falciparum malaria in western Kenya. Trop Med Int Health. 2009;14:294-300.

50. Rwagacondo CE, Karema C, Mugisha V, Erhart A, Dujardin JC, Overmeir CV, et al. Is amodiaquine failing in Rwanda? Efficacy of amodiaquine alone and combined with artesunate in children with uncomplicated malaria. Trop Med Int Health. 2004;9:1091-8.

51. Dorsey G, Staedke S, Clark TD, Njama-Meya D, Nzarubara B, Maiteki-Sebuguzi C, et al. Ombination therapy for uncomplicated falciparum malaria in Ugandan children: a randomized trial. Jama. 2007;297:2210-9.

52. Zongo I, Dorsey G, Rouamba N, Dokomajilar C, Séré Y, Rosenthal PJ, et al. Randomized comparison of amodiaquine plus sulfadoxine-pyrimethamine, artemether-lumefantrine, and dihydroartemisinin-piperaquine for the treatment of uncomplicated Plasmodium falciparum malaria in Burkina Faso. Clin Infect Dis. 2007:45:1453-61.

53. Schramm B, Valeh P, Baudin E, Mazinda CS, Smith R, Pinoges L, et al. Efficacy of artesunate-amodiaquine and artemether-lumefantrine fixed-dose combinations for the treatment of uncomplicated Plasmodium falciparum malaria among children aged six to 59 months in Nimba County, Liberia: an open-label randomized non-inferiority trial. Malar J. 2013;12:251.

54. Whitty CJ, Staedke SG. Artemisinin-based combination treatment for malaria in Africa: no perfect solutions. Clin Infect Dis. 2005;41:1087-8.

55. Plucinski MM, Talundzic E, Morton L, Dimbu PR, Macaia AP, Fortes F, et al. Efficacy of artemether-lumefantrine and dihydroartemisinin-piperaquine for the treatment of uncomplicated malaria in children in Zaire and Uíge Provinces, Angola. Antimicrob Agents Chemother. 2015;59:437-43.

56. WHO. World malaria report 2008. Geneva: World Health Organization; 2008.

57. Haug S, Lakew T, Habtemariam G, Alemayehu W, Cevallos V, Zhou Z, et al. The decline of pneumococcal resistance after cessation of mass antibiotic distributions for trachoma. Clin Infect Dis. 2010;51:571-4.

58. van Eijk AM, Terlouw DJ. Azithromycin for treating uncomplicated malaria. Cochrane Database Syst Rev. 2011;2, CD006688.

59. Parasite Clearance Estimator (PCE) [https://www.wwarn.org/pce/].

60. Flegg JA, Guerin PJ, White NJ, Stepniewska K. Standardizing the measurement of parasite clearance in falciparum malaria: the parasite clearance estimator. Malar J. 2011;10:339.

61. Price RN, Nosten F, Luxemburger C, Ter Kuile F, Paiphun L, Price R, et al. Effects of artemisinin derivatives on malaria transmissibility. Lancet. 1996;347:1654-8.

62. Mens PF, Sawa P, van Amsterdam SM, Versteeg I, Omar SA, Schallig H, et al. A randomized trial to monitor the efficacy and effectiveness by QT-NASBA of artemether-lumefantrine versus dihydroartemisinin-piperaquine for treatment and transmission control of uncomplicated Plasmodium falciparum malaria in western Kenya. Malar J. 2008;7:237.

63. Adjuik M, Babiker A, Garner P, Olliaro P, Taylor W, White N. Artesunate combinations for treatment of malaria: meta-analysis. Lancet. 2004;363:9-17.

64. Omari AA, Gamble C, Garner P. Artemether lumefantrine for uncomplicated malaria: a systematic review. Trop Med Int Health. 2004;9:192-9.

65. White N. Preventing antimalarial drug resistance through combinations. Drug Resist Updat. 1998;1:3-9.

66. Bell DJ, Wootton D, Mukaka M, Montgomery J, Kayange N, Chimpeni P, et al. Measurement of adherence, drug concentrations and the effectiveness of artemether-lumefantrine, chlorproguanil-dapsone or sulphadoxine-pyrimethamine in the treatment of uncomplicated malaria in Malawi. Malar J. 2009:8:204.

67. Checchi F, Piola P, Fogg C, Bajunirwe F, Biraro S, Grandesso F, et al. Supervised versus unsupervised antimalarial treatment with six-dose artemether-lumefantrine: pharmacokinetic and dosage-related findings from a clinical trial in Uganda. Malar J. 2006:5:59.

68. Piola P, Fogg C, Bajunirwe F, Biraro S, Grandesso F, Ruzagira E, et al. Supervised versus unsupervised intake of six-dose artemether-lumefantrine for treatment of acute, uncomplicated Plasmodium falciparum malaria in Mbarara, Uganda: a randomised trial. Lancet. 2005;365:1467-73.

69. Sisowath C, Strömberg J, Mårtensson A, Msellem M, Obondo C, Björkman A, et al. In vivo selection of Plasmodium falciparum pfmdr1 $86 \mathrm{~N}$ coding alleles by artemether-lumefantrine (Coartem). J Infect Dis. 2005;191:1014-7.

70. Faye B, Offianan AT, Ndiaye JL, Tine RC, Touré W, Djoman K, et al. Efficacy and tolerability of artesunate amodiaquine (Camoquin plus ${ }^{\oplus}$ ) versus artemether lumefantrine (Coartem ${ }^{\oplus}$ ) against uncomplicated Plasmodium falciparum malaria: multisite trial in Senegal and Ivory Coast. Trop Med Int Health. 2010;15:608-13.

71. Schramm B, Valeh P, Baudin E, Mazinda CS, Smith R, Pinoges L, et al. Tolerability and safety of artesunate-amodiaquine and artemetherlumefantrine fixed dose combinations for the treatment of uncomplicated Plasmodium falciparum malaria: two open-label, randomized trials in Nimba County. Liberia Malar J. 2013;12:250

72. O'Dempsey T, McArdle T, Laurence B, Lamont A, Todd J, Greenwood B. Overlap in the clinical features of pneumonia and malaria in African children. Trans R Soc Trop Med Hyg. 1993;87:662-5.

73. Egunsola O, Oshikoya KA. Comparative safety of artemether-lumefantrine and other artemisinin-based combinations in children: a systematic review. Malar J. 2013;12:385. 
74. Conrad MD, Bigira V, Kapisi J, Muhindo M, Kamya MR, Havlir DV, et al. Polymorphisms in K13 and Falcipain-2 associated with artemisinin resistance are not prevalent in Plasmodium falciparum isolated from Ugandan Children. PLoS One. 2014;9:e105690.

75. Ghansah A, Amenga-Etego L, Amambua-Ngwa A, Andagalu B, Apinjoh T, Bouyou-Akotet $\mathrm{M}$, et al. Monitoring parasite diversity for malaria elimination in sub-Saharan Africa. Science. 2014;345:1297-8.

Submit your next manuscript to BioMed Central and take full advantage of:

- Convenient online submission

- Thorough peer review

- No space constraints or color figure charges

- Immediate publication on acceptance

- Inclusion in PubMed, CAS, Scopus and Google Scholar

- Research which is freely available for redistribution 